\title{
CONTRIBUTION A L'ÉTUDE DE LA RÉTENTION ET DE L'ÉPURATION PULMONAIRE D'OXYDE DE PLUTONIUM
}

\author{
MISE EN ÉVIDENCE DE LA PHAGOCYTOSE \\ PAR LES MACROPHAGES ALVÉOLAIRES IN VIVO ET IN VITRO
}

\author{
(manuscrit reģu le s juin 1967 )
}

\section{I - INTRODUCTION}

De nombreuses expérimentations ont montré la toxicité du plutonium et les risques encourus par les personnes travaillant ce métal. Son oxyde particulièrement insoluble présente, après inhalation, une rétention pulmonaire longue que le C.I.P.R. estime être de soo jours pour l'homme après une exposition unique. Expérimentalement ( $\mathrm{I}$ ) une leucopénie accompagne les troubles respiratoires : fibrose sévère et formations métaplasiques qui entrainent la mort. La migration d'une fraction du plutonium a lieu vers les ganglions péribronchiques et médiastinaux dans lesquels il s'accumule et induit des transformations progressives aboutissant à du tissu fibreux. L'épuration spontanée et le stockage tissulaire étudiés par MARrow (2) confirment ces faits.

Le plutonium, comme la silice ou le béryllium, pour ne citer que ces exemples, offre à la toxicologie expérimentale un champ d'exploration immense, car si les phénomènes de clearance alvéolaire donnent lieu a de nombreuses études, beaucoup reste à faire pour que l'épuration spontanée soit secourue par une épuration provoquée dont nous ne connaissons pas encore les facteurs favorisants.

\section{II - DESCRIPTION ET ROLE DU MACROPHAGE ALVÉOLAIRE}

Le comité II de la Commission Internationale de Protection contre les Radiations a suscité une étude de la "dynamique pulmonaire ». Le rapport (3) fait le point des connaissances acquises et des hypothèses de travail, en particulier dans le domaine de l'épuration pulmonaire et de l'endocytose alvéolaire; il conclut au rôle majeur joué par ce phénomène, mais souligne la nécessité d'expérimentations complémentaires.

Dès 1959, les travaux de LA BELLE (4) ont montré l'importance du pouvoir phagocytaire des cellules alvéolaires libres, "les macrophages ", et leur rôle dans l'épuration a été confirmé tout au long du "Second International Symposium on inhaled Particles and Vapours » tenu à Cambridge en 1965 . Pour les poussières fortement retenues et en particulier pour l'oxyde de plutonium, la phase d'épuration alvéolaire qui est lente et dominante, dépend directement des caractéristiques physico-chimiques de la poussière, du nombre des macrophages ainsi que de leur état fonctionnel.

Au niveau des alvéoles pulmonaires, on trouve des cellules type histiocytes; le terme " macrophage alvéolaire » est controversé quant à ce qu'il désigne. Pour Karrer ( 5 ), Policard et CoLLET (6), il est utilisé pour désigner les cellules libres dans les alvéoles du poumon normal, participant à l'assainissement des espaces alvéolaires en conditions physiologiques, aussi bien que dans des conditions pathologiques, en phagocytant les poussières inhalées, les débris de cellules, les bactéries, etc.

Ces macrophages alvéolaires sont des cellules libres, arrondies, caractérisées par la présence d'une membrane ondulante qui leur confère une grande mobilité. Ils présentent une série de caractères généraux :

- richesse en réticulum endoplasmique lisse,

- abondance de mitochondries,

- grand développement de l'appareil de Golgi.

Enfin un caractère très spécifique du macrophage alvéolaire est sa richesse en lysosomes et donc corrélativement en hydrolases (glucosidase, glycuronidase, ribonucléase et surtout phosphatase acide).

Les lysosomes sont fortement concernés dans la phagocytose, Policard (7), CoHn (8), Weissmann (9), Cattan (ir) en ont décrit toute l'importance. Les membranes des lysosomes 
fusionnent avec celles des vacuoles phagocytaires contenant le matériel capté. Les hydrolases acides sont déversées dans la vacuole, elle prend alors le nom de phagosome.

Parmi les particules solides prises par la cellule phagocytaire, celles qui ne sont pas toxiques restent indéfiniment à l'intérieur, celles qui le sont comme la silice vont provoquer la mort de la cellule, les enzymes s'échappent rapidement dans le cytoplasme alentour; dans ce cas l'acide silicique réagit avec les membranes des phagosomes (ro). Les particules de silice mises en liberté par la mort de la cellule sont reprises par d'autres cellules, le processus continue et donne naissance à la formation de tissu fibreux.

III - TeChNiques UTILISÉES EN VUe DE LA Mise EN ÉVIDENCE DE LA PHAGOCytose DE L'OXYDE DE PLUTONIUM PAR LE MACROPHAGE ALVÉOLAIRE

Dans le cas particulier de l'oxyde de plutonium dont la radioactivité associée à l'état particulaire peut induire dans la cellule des phénomènes de radiolyse, il semble intéressant d'étudier le devenir du macrophage qui assurera le transport du plutonium jusqu'au niveau ganglionnaire.

Dans le cadre de cette étude nous utilisons une série de techniques dont nous donnerons ici les grandes lignes, notre but étant de comparer le comportement des macrophages alvéolaires dans les deux cas suivants :

a) Les macrophages sont recueillis après sacrifice des animaux précédemment empoussiérés.

b) Les macrophages sont récoltés à partir d'animaux sains et mis en contact "in vitro " avec des poussières de mêmes caractéristiques granulométriques.

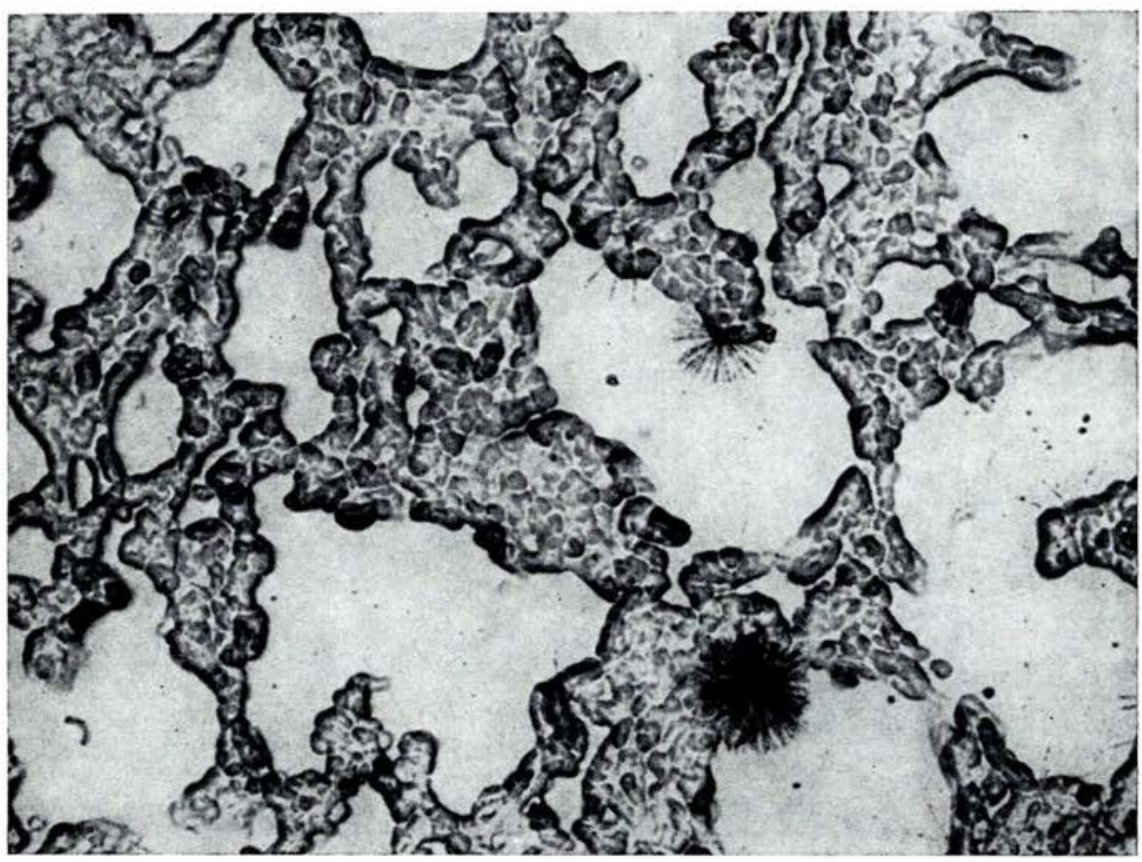

FIG. I.

\section{III.I. Tecbnique d'empoussierage des animaux}

Les animaux soumis à l'empoussiérage sont des rats d'un poids moyen de $200 \mathrm{~g}$, le matériel a été décrit dans un précédent rapport (I6). L'oxyde de plutonium utilisé a un diamètre moyen de 0,50 micron, l'écart type est de 1,50 . 


\section{III.2. Prélèvement et survie des macrophages}

LA Belle et Brieger (12) ont montré qu'il est possible de récolter les macrophages alvéolaires vivants afin de les étudier hors de l'organisme. Leur technique modifiée par MYRVICK (I3) consiste, après sacrifice de l'animal à dégager le bloc cardio-pulmonaire dont l'extérieur est lavé avec du sérum physiologique et à injecter dans la trachée un volume variable de solution de Hanks à $37^{\circ}$, selon la grosseur de l'animal. Après un massage délicat du poumon le liquide de lavage est aspiré et centrifugé. Le culot cellulaire est remis en suspension dans un volume connu de liquide de Hanks sur lequel est effectuée la numération des macrophages.

La mise en service est effectuée selon les techniques de Vorsın (14), les cellules récoltées et comptées sont mises en suspension dans le liquide de Earle à l'hydrolysat de lactalbumine additionné de sérum de veau et d'antibiotiques. Les cellules continuent à vivre et des prélèvements à des temps déterminés permettent d'effectuer sur elles des mesures biochimiques, de tester leur comportement phagocytaire et de les examiner en microcinématographie au moyen de la cellule de BARSKI (Is).

\section{III.3. Tecbnique d'empoussiérage "in vitro" des cellules}

Immédiatement après la récolte des macrophages, la mise en contact se fait par addition à une suspension contenant $10^{6}$ cellules par $\mathrm{ml}$, de l'oxyde de plutonium calibré et dosé. Au bout de deux heures, la préparation est centrifugée et les macrophages isolés sont étudiés par autoradiographie, comme dans le cas d'empoussiérage in vitro.

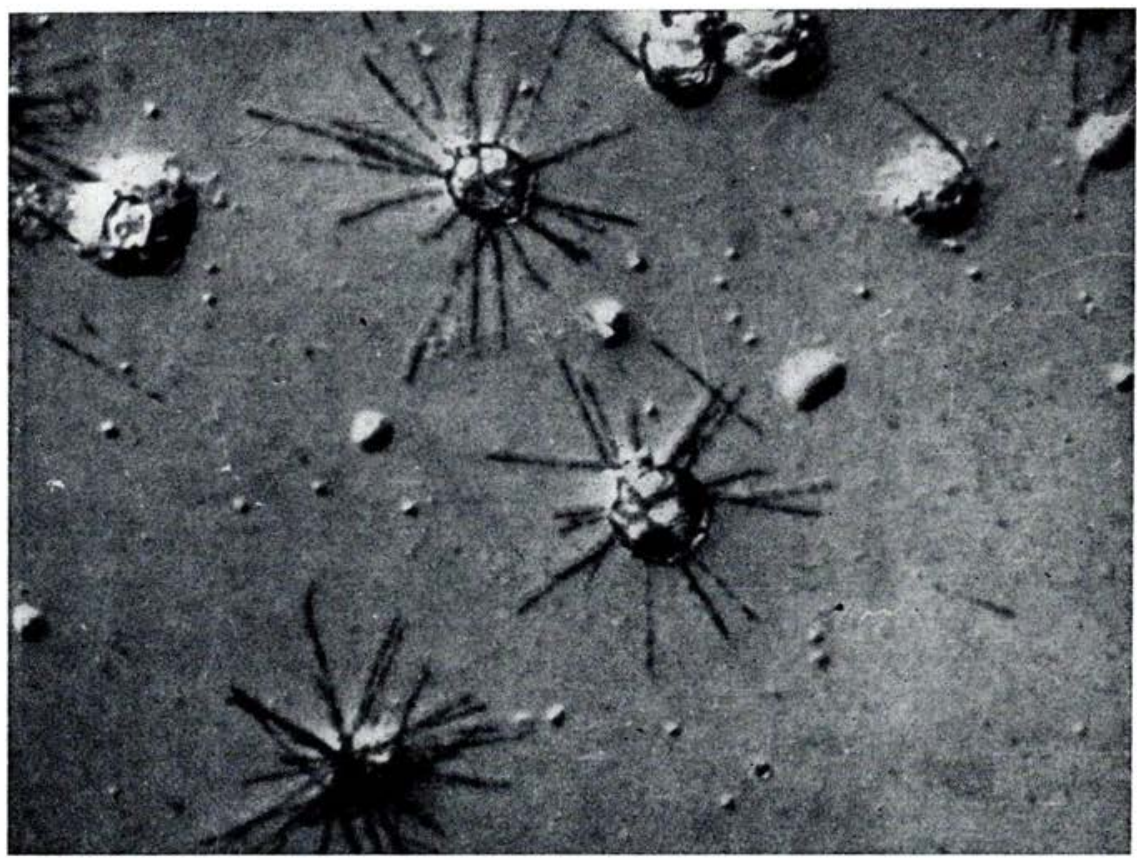

FIG. 2.

\section{III.4. Etude autoradiographique des cellules}

L'autoradiographie est effectuée sur des émulsions nucléaires Ilford, type $\mathrm{K}_{2}$, d'épaisseur 25 microns, sensibles aux rayonnements $\alpha$.

Dans l'obscurité, un étalement par frottis sur l'émulsion est fait à partir d'une petite quantité de suspension concentrée de macrophages. Le frottis est séché à l'air, les cellules sont fixées 
pendant 2 minutes à l'alcool méthylique. Après séchage les lames sont laissées à l'obscurité pendant 24 heures à $4^{\circ}$ et développées selon la méthode classique.

A l'observation microscopique en contraste de phase, les cellules sont visibles et les poussières d'oxyde de plutonium sont matérialisées par une "étoile", chaque branche de l'étoile correspondant à la désintégration d'un atome, la particule $\alpha$ émise donnant une trace rectiligne dans l'émulsion photographique.

Les macrophages qui ont phagocyté une ou plusieurs poussières apparaissent en superposition avec une ou plusieurs "étoiles" (fig. 2).

Parallèlement à l'étude des macrophages, nous avons mis en évidence la rétention alvéolaire d'oxyde par autoradiographie de coupes histologiques de poumon. Après sacrifice de l'animal un fragment de poumon est traité par la méthode histologique classique et les coupes obtenues sont recouvertes d'une émulsion gel Ilford Ko, l'examen microscopique montre en superposition le tissu pulmonaire et les étoiles correspondant à chaque poussière (fig. I).

\section{IV - Conclusion}

L'exploration microscopique des autoradiographies obtenues permet de mettre en évidence la phagocytose des particules de plutonium par les macrophages.

Dans le cas des macrophages mis en contact in vitro, on obtient deux types de figures étoilées, les "étoiles" associées aux macrophages, et les " étoiles» isolées correspondant à des poussières libres.

Le pourcentage des cellules ayant phagocyté est inférieur à celui observé après empoussiérage in vivo, dans ce dernier cas nous n'avons pas remarqué d' "étoile " correspondant à des poussières quelle que soit la durée de survie des animaux.

Parallèlement, l'examen morphologique des macrophages montre une altération notable des cellules examinées au bout d'un temps long de survie.

Ces premiers résultats nous ont permis d'entreprendre des études métaboliques et ultra structurales de ces cellules dont les résultats feront l'objet d'un prochain rapport.

Les macrophages alvéolaires constituent un matériel de travail particulièrement intéressant parce qu'ils jouent un rôle capital dans l'épuration pulmonaire, qu'ils se prêtent facilement à une survie hors de l'organisme et que parallèlement aux leucocytes, leurs propriétés phagocytaires permettent d'envisager une étude de l'action des rayonnements par irradiation intra-cellulaire. Il est donc important de chiffrer leur comportement physiologique en présence de poussières aussi particulières que l'oxyde de plutonium.

Michèle Kunzle-Lutz, Daniel Nolibe, Huguette Rouvroy.

C.E.A. Centre d'Études de Bruyères-le-Chatel. $\mathrm{BP}^{\circ}{ }^{\circ}$ 6I $-92-\mathrm{MONTROUGE}$

\section{BIBLIOGRAPHIE}

(I) Clarke. W.J., Bair, W.J. Plutonium inhalation studies. VI : Pathologic effects of inhaled plutonium particles in dogs, Health Pbysics, 1964, Vol. 10, 391-398.

(2) Morrow, P.E., Cibb, F.R., Davies, H., Mitola, J., Wood, D., Wraight, N., Cambael, H.S. The retention and fate of inhaled plutonium dioxide in dogs, Health Pbysics, 1967, Vol. 13, II3-133.

(3) TASK group on LUNG exposures. Deposition and retention models for internal dosimetry of the human respiratory tract, Health Pbysics, 1966, Vol. 12, 173-207.

(4) La Belle, C.W., Brieger H., A.M.A. Arch. Industr. Hlth 1959, 20, 100-105.

(s) KARRER. H.E. The ultrastructure of mouse lung : the alveolar macrophage, J. of Biophysical and Biocbemical Cytology, 1958, Vol. 4.

(6) Policard, A., Collet, A., Martin, J.C., Pregermain, S., Revet, C. Etude infrastructurale des macrophages alvéolaires libres isolés du poumon chez le cobaye. C.R. Acad. des $S_{c .}, 1963$, Vol. $256, \mathrm{n}^{\circ} 16$.

(7) Collet, A., Martin, J.C., Ruet-Normand, C., Policard, A. Recherches infrastructurales sur l'évolution des macrophages alvéolaires et leurs réactions aux poussières minérales. in : Inhaled Particles and vapours. Cambridge, 1967, Vol. 2. 
(8) CoHn, Z.A., WIENER, E. The particulate hydrolases of macrophages. I : Comparative enzymology, isolation and properties. J. Exp. Med., $1963,118$.

(9) Werssmann, G. Lysosomes, Blood, 1964, Vol. 24, 594-606.

(ro) Allison A.C. The lysosome, Discovery, 1965, Vol. 26, no 8.

(ir) Catran, A. Les lysosomes, Revue Franfaise d'Etudes Cliniques et Biologiques, 1966, Vol. II, $\mathrm{n}^{\circ} 3$, p. 235 .

(12) La Belle, C.W., Brieger, H. in : Inhaled vapours and particles, Oxford, 196r, p. 356.

(13) Myrvick, Q.N., Leave E.S., Fariss, B. Studies on pulmonary alveolar macrophages from the normal rabbit : a technique to procure them in a high state of purity, J. Immunol,, 1961, 86-1 28.

(i4) Voisin, C., Guillaume, J., Van-Moorleghem, C., Aerts, C. Selection et mise en service in vitro de macrophages alvéolaires de cobaye, Annales de l'Institut de Lille, Vol. I4, 1963 .

(IS) BARski, G., Robineaux, R. Chambre à perfusion démontable et stérilisable pour culture de tissus de longue durée, Annales de l'Institut Pasteur, 1956, Vol. 90, p. 514.

(16) Lutz, M., Rouvrox, H. Description d'un dispositif permettant la contamination d'animaux de laboratoire par inhalation d'aérosols radioactifs, Rapport CE $A$ R 3086.

\section{NOTE D'INFORMATION}

\section{LABORATOIRES ASSOCIÉS DE PHYSIQUE DES AÉROSOLS}

Trois laboratoires français se sont groupés pour collaborer à l'étude des problèmes physiques posés par les aérosols : le laboratoire d'électricité atmosphérique et de physique des nuages de la Faculté des Sciences de Paris (Professeur Bricard), le Service Technique d'Etudes de Protection du Commissariat à l'Energie Atomique (MM. Pradel et Brllard) et le Centre de Physique Atomique et Nucléaire de la Faculté des Sciences de Toulouse (Professeurs Blanc et Fontan). Ce groupement de laboratoires, placé sous la direction scientifique du Professeur Bricard a décidé de prendre le nom de "Laboratoires associés de Physique des Aérosols». La correspondance est à adresser à :

Laboratoires associés de Physique des Aérosols, Professeur Bricard, 3, boulevard Pasteur, 75 - Paris-15 ${ }^{e}$. 